\title{
Modelagem Manual e Digital de Personagens como Ferramenta Lúdica e Auxiliar no Ensino-Aprendizagem de Anatomia Humana
}

\author{
Arthur Eduardo de Oliveira Morais'1, Vitória Facundo Macedo \\ ${ }^{1}$ Sistemas e Mídias Digitais - Universidade Federal do Ceará (UFC) \\ R. Pernambuco, 2986-3030 - Demócrito Rocha, 60440-140 - Fortaleza - CE - Brasil \\ arthuredu15@gmail.com, vitoriafacundomegmail.com
}

\begin{abstract}
This article aims to demonstrate the process of application of the workshop 'Manual Modeling of Characters', that results in the production of anatomical models made in modeling clay or plasticine. In addition, demonstrate the advantages of working playfulness in the process of teaching more complex content and the potential of the proposed methodology for teaching students with hearing and visual impairment.. The human anatomy is especially impaired by the application of traditional methods of teaching that are based on memorization in an arbitrary way. In this way, this work seeks to encourage the replication of the process demonstrating the adopted procedure and the possibilities of expansion for the digital medium.
\end{abstract}

Resumo. Este artigo tem por objetivo demonstrar o processo de aplicação da oficina 'Modelagem Manual de Personagens', que resulta na produção de modelos anatômicos feitos em massa de modelar ou plastilina. Além disso, demonstrar as vantagens de se trabalhar a ludicidade no processo de ensino de conteúdos de maior complexidade e o potencial da metodologia proposta para o ensino de alunos com deficiência auditiva e visual. A anatomia humana é especialmente prejudicada diante da aplicação dos tradicionais métodos de ensino que têm como base a memorização de forma arbitrária. Desta forma, este trabalho busca incentivar a replicação do processo demonstrando o procedimento adotado e as possibilidades de expansão para o meio digital.

\section{Introdução}

Apesar das divergências sobre qual seria o melhor método de ensino, atualmente há uma crescente concordância a respeito das falhas do tradicional método de Aprendizagem Mecânica (AUSUBEL, 1978) em transmitir conteúdo aos alunos de forma efetiva. Esse método consiste na incorporação de um conhecimento novo de forma arbitrária, onde o 
aluno precisa aprender sem antes entender do que se trata ou compreender o significado do porquê (BRAATHEN, 2012). Esse conceito se traduz para a nossa realidade através de professores que costumam escrever na lousa uma série de definições e conceitos e esperam que os alunos anotem e memorizem de forma automática, o que resulta em conteúdos soltos ou ligados à estrutura mental de forma fraca (AUSUBEL, 1978).

Alguns conteúdos são especialmente prejudicados por esse método de abordagem, como é o caso da Anatomia Humana. Sua complexidade é comumente atribuída a grande quantidade de conceitos e estruturas (SALBEGOI et al, 2015), podendo tornar o processo de aprendizagem exaustivo caso não haja uma estratégia de ensino adequada. Outro obstáculo relatado pelos estudantes dessa disciplina é a dificuldade na visualização de estruturas corporais, comumente representadas por imagens bidimensionais nos livros didáticos (SILVEIRA e IDERIHA, 2013). Em função disso, a visualização e contato direto com uma representação tridimensional representa uma forte aliada no processo de aprendizagem.

Ao tratar do tema tecnologia aplicado na educação, é importante explicitar seu significado. 'Tecnologia' tem origem no grego "tekhne" que significa "técnica, arte, ofício" (Dicionário Etimológico, 2019). Também pode ser definida de acordo com o dicionário Michaelis (2015) como um conjunto de processos, métodos, técnicas e ferramentas relativos a arte, indústria, educação etc. Aprofundando nesse significado percebemos que a aplicação da tecnologia como ferramenta de aprendizado vai muito além de apenas introduzir recursos digitais nas salas de aula, podendo também acontecer através da inserção de novos métodos de ensino prático. Aliado a isso a teoria da Aprendizagem Significativa proposta por Ausubel (1963) incentiva os docentes na proposição de atividades que favoreçam o aprendizado, criando momentos capazes de potencializar a construção do conhecimento.

A Educação Lúdica consiste em um recurso que insere jogos e brincadeiras no auxílio da transmissão do conteúdo (SANT'ANNA e NASCIMENTO, 2011). Atualmente, a aplicação desse método como base de processos de aprendizagem está em voga. Porém é importante ressaltar que ele vai além da inclusão de jogos relacionados com os temas abordados em sala de aula. Atividades Lúdicas são aquelas que possibilitam momentos de prazer, entrega e integração dos envolvidos, propiciando uma experiência de plenitude, em que nos envolvemos por inteiro, estando flexíveis e saudáveis (LUCKESI, 2003).

No curso de Sistemas e Mídias Digitais (SMD) da Universidade Federal do Ceará (UFC) existe a possibilidade de entrelaçar diferentes ramos do conhecimento na proposta de soluções para problemáticas apresentadas ao longo das experiências dentro e fora da universidade. Dentre estas experiências está o Projeto de Extensão da UFC, Media Day, que tem como proposta levar conhecimentos de diferentes áreas do curso para os alunos de Escolas Estaduais de Educação Profissional (EEEP) localizadas em cidades do Ceará. Nessas unidades é ofertado o Ensino Médio integrado à Educação Profissional, os alunos têm em média entre 14 e 17 anos de idade e seguem o currículo da base nacional comum além da formação profissional. 
Para o Projeto foi desenvolvida a oficina 'Modelagem Manual de Personagens' que tem como objetivo auxiliar no processo de ensino-aprendizagem dos alunos na compreensão da anatomia humana através da concepção de modelos tridimensionais de plastilina ou massinha de modelar, com enfoque na estrutura óssea da cabeça humana. Promovendo a absorção do conteúdo, exploração da criatividade e a ludicidade associada ao processo de dar forma a um personagem significativo para o contexto pessoal do indivíduo.

Como recurso complementar, os alunos são encorajados a replicar o processo manual por meio de ferramentas digitais. Isso ocorre através da indicação de softwares gratuitos de modelagem tridimensional, como Blender e Sculptris, ou aqueles com versões gratuitas para teste, como Zbrush. Além disso, são indicados tutoriais online para facilitar a modelagem. Com essa transição para o digital, os estudantes podem expandir as possibilidades de aplicação da obra para outras mídias e aplicações, como a animação.

\section{Modelagem Manual como Base do Processo}

O método de modelagem manual traz como principal diferencial a possibilidade de estender o canal de aprendizado entre o aluno e o conteúdo para o meio tátil. Uma vez que tradicionalmente essa comunicação ocorre de forma oral e visual. A exploração da memória sensorial no processo de aprendizagem é um recurso fundamental pois além de fortalecer a memorização do conteúdo, dá margem para a inclusão de diferentes tipos de alunos, fomentando a acessibilidade como pilar do processo de ensino. No caso da oficina proposta, a memória tátil beneficiaria diretamente deficientes visuais.

Além disso, alunos com surdez também são beneficiados, pois o método representa uma alternativa ao ensino oral-auditivo. Mesmo com o auxílio de intérpretes, os alunos têm dificuldade para entender o que o professor está explicando (BELTRAMIN; GÓIS, 2012). Aliado a isso, a comunidade surda requer especial atenção no uso de recursos visuais a serem aplicados no seu processo de ensino-aprendizagem (CARVALHO, 2016).

Sobre aprendizagem significativa, David Ausubel define:

“O processo ideal ocorre quando uma nova ideia se relaciona aos
conhecimentos prévios do indivíduo. Motivado por uma situação que faça
sentido, proposta pelo professor, o aluno amplia, avalia, atualiza e
reconfigura a informação anterior, transformando-a em
nova.".(AUSUBEL, 1963)

Esse processo é adotado na oficina, tendo a proposta representada na construção do modelo da figura humana, o aluno avalia a escultura obtida e reconfigura as definições de cada parte corporal à medida que reconfigura a forma do seu objeto, para que esse se adeque ao seu personagem.

O método de modelagem adotado consiste na blocagem das partes, isto é, modelar separadamente cada um dos elementos da estrutura corporal. Tal processo facilita a introdução das nomenclaturas científicas adequadas ao estudo da anatomia e, simultaneamente, o próprio ato de modelar, uma vez que após a confecção de cada componente o aluno deve mesclá-los para formar o todo. 
O incentivo para estender a prática manual para o meio digital se dá para facilitar o contato constante do aluno com sua obra e estimular a manutenção do conhecimento adquirido durante a oficina, uma vez que o material utilizado na prática é limitado e reutilizado, torna-se inviável a aquisição do modelo físico. Dessa forma, o aluno pode visualizar na tela determinada parte do corpo modelada através de diferentes ângulos, da mesma forma realizada com o modelo físico. Esse tipo de interação ocorrendo no meio virtual se relaciona, ainda que de forma primária, com a definição de Moore (1995) para Ambientes de Realidade Virtual Desktop, caracterizada como a extensão de uma interação envolvendo imagens tridimensionais acrescida de uma experiência de interação multimídia. Sem necessariamente ser considerada imersiva (MOORE, 1995). Tais ambientes representam ferramentas poderosas no processo de aprendizagem, uma vez que, dentro de uma das interpretações do método de Aprendizagem Construtivista atribuído a Piaget (MCINERNEY AND MCINERNEY, 1994; SLAVIN, 1994), propiciam o engajamento através de um processo de exploração ativa (DALGARNO, 2002). Dessa forma, indo adiante do que acontece durante a oficina, onde é necessária a presença de um tutor, na passagem para o meio digital, e consequentemente na exploração do modelo anatômico, estimula-se a independência do aluno no seu próprio processo de aprendizagem.

Existe ainda uma grande vantagem em em expandir os modelos tridimensionais para o ambiente virtual: uso de impressoras 3D. Essas se apresentam como fortes aliadas para alunos com deficiência visual, pois permite a confecção de peças com diferentes relevos, texturas e contrastes. A diferenciação de superfícies do tipo liso/áspero, fino/espesso, propiciam a distinção adequada, expandindo as possibilidades de partes anatômicas que podem ser reproduzidas (FROSCH, 2018). O professor e pesquisador Renato Frosch desenvolveu um projeto de modelagem 3D para a construção de modelos pedagógicos acessíveis e, sabendo da escassez de materiais voltado à impressão 3D, disponibiliza todos os materiais produzidos de maneira gratuita na internet1, possibilitando a autonomia dos educadores na utilização dos recursos. Além disso, por se tratar de uma matéria prima de baixo custo, o material produzido pode ser distribuído para os alunos levarem para casa e usarem como material de estudo, diferente do que ocorre na prática com a plastilina.

\section{Metodologia}

Os materiais básicos para a realização da oficina são massa de modelar, preferencialmente plastilina de cor única e alguma ferramenta para realizar marcações na peça, como lápis ou palitos. Como material complementar, existem estecas ou espátulas específicas voltadas para modelagem manual e que, apesar de não serem fundamentais para a realização do processo, adicionam um nível de refinamento que contribui diretamente para a fidelidade do produto final.

Inicialmente, é passada uma amostra do material para que os alunos tenham um primeiro contato e possam explorá-lo livremente. Em seguida, cerca de $200 \mathrm{~g}$ de plastilina é fornecida para cada aluno ou, para aqueles que sentirem-se mais confortáveis em 
trabalhar em equipe, essa porção é fornecida para a modelagem de uma única peça por um grupo de até 3 pessoas.

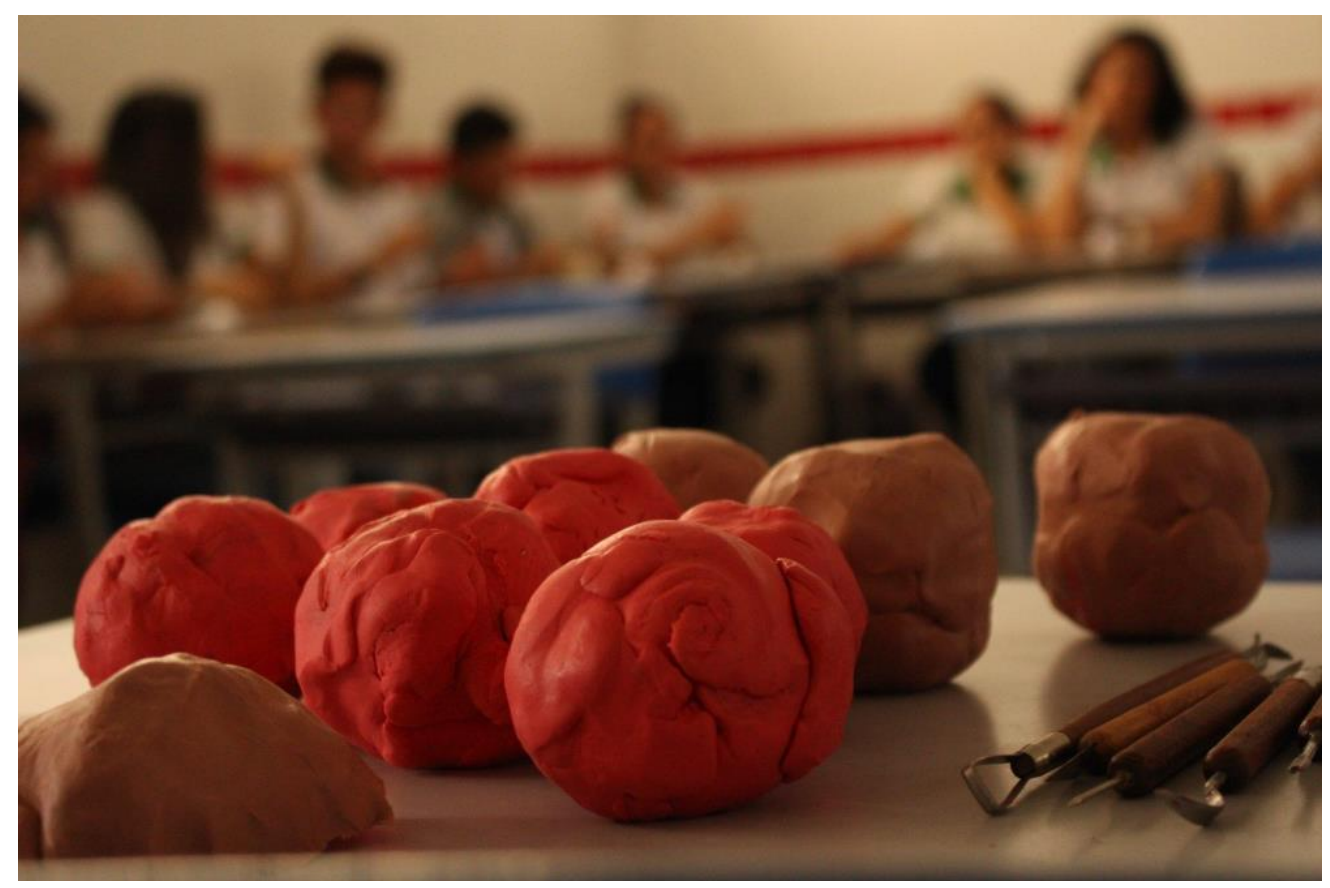

Figura 1 - Material disponibilizado

Para inserir a ferramenta lúdica ao procedimento os alunos são orientados a escolher um personagem de livro, filme ou animação de seu interesse para servir de modelo, tendo como única exigência que sejam humanos ou com traços humanóides. Se possível, pesquisar imagens de referência para guiá-los no processo de modelagem. Também é oferecida a possibilidade de ser um personagem criado pelo próprio aluno. No passo seguinte, são dadas as primeiras definições teóricas anatômicas, juntamente com imagens bidimensionais das partes que serão trabalhadas. O processo é iniciado com as partes mais amplas da estrutura óssea da cabeça humana: Crânio e Maxilar Inferior. Para a replicação com a plastilina os alunos observam um modelo, produzido na hora, de cada uma das etapas do processo.

A priori, nessa primeira parte, incentivamos o aluno a manter a fidelidade com a figura humana, independente do personagem. Isso se justifica pela falta de familiaridade com o processo manual e para não associar equivocadamente uma forma humana anatomicamente definida à modificação realizada através do exagero ou deslocamento dessa mesma forma na construção de seu personagem.

Ao longo da oficina é imprescindível que as partes trabalhadas sejam referidas conforme os termos estudados na parte teórica. Isso auxilia no processo de absorção do conteúdo. Outro processo que é contínuo e deve ser incentivado é a comparação entre modelo construído, modelo anatômico bidimensional e personagem escolhido. Apesar de as adaptações à figura escolhida só ocorrerem na segunda parte da oficina, é importante que o aluno tenha sempre em mente seu objetivo, de modo que possa estar à todo momento estudando sua obra e quais serão as mudanças de dimensão e posicionamento que precisarão ser feitas na etapa de adaptação. 


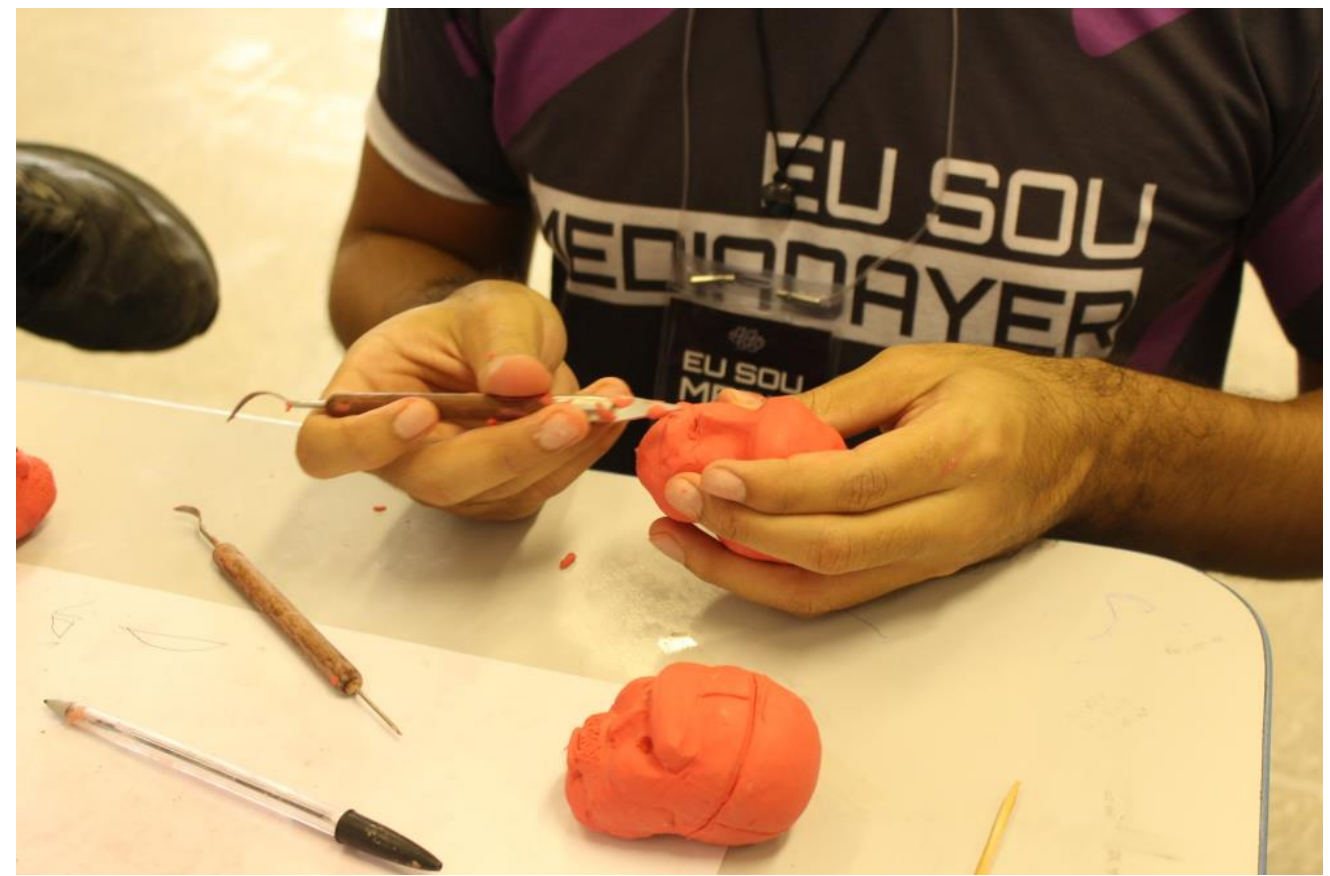

Figura 2 - Demonstração do processo

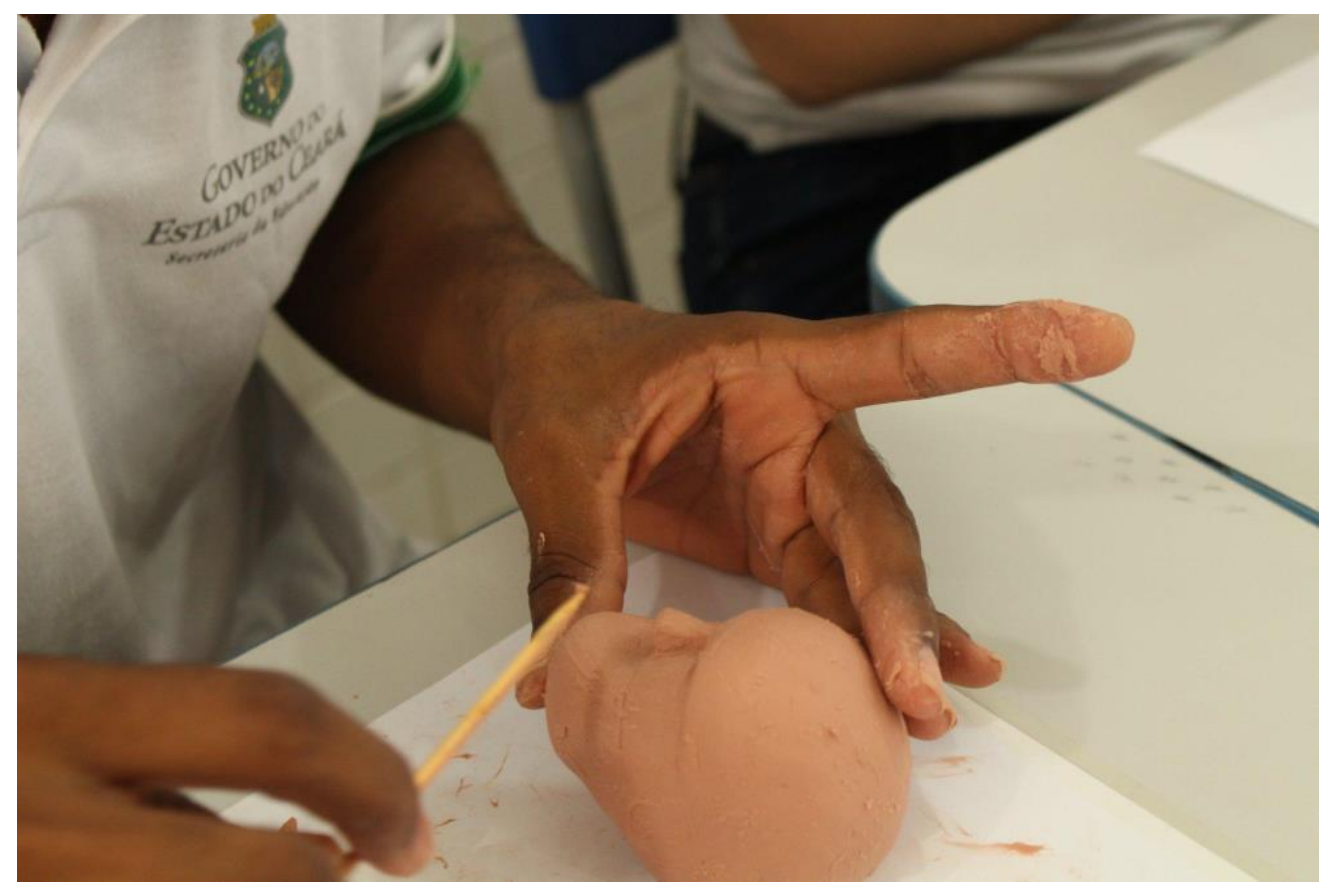

Figura 3 - Replicando o modelo

Uma vez que a cabeça humana esteja completa, os alunos podem explorar a criatividade na remodelagem da peça, acrescentando características de seu personagem referencial. Nessa etapa da prática são disponibilizados objetos diversos e diferentes cores do material utilizado na modelagem. Aqui, o objetivo é deixá-los o mais livre possível. 


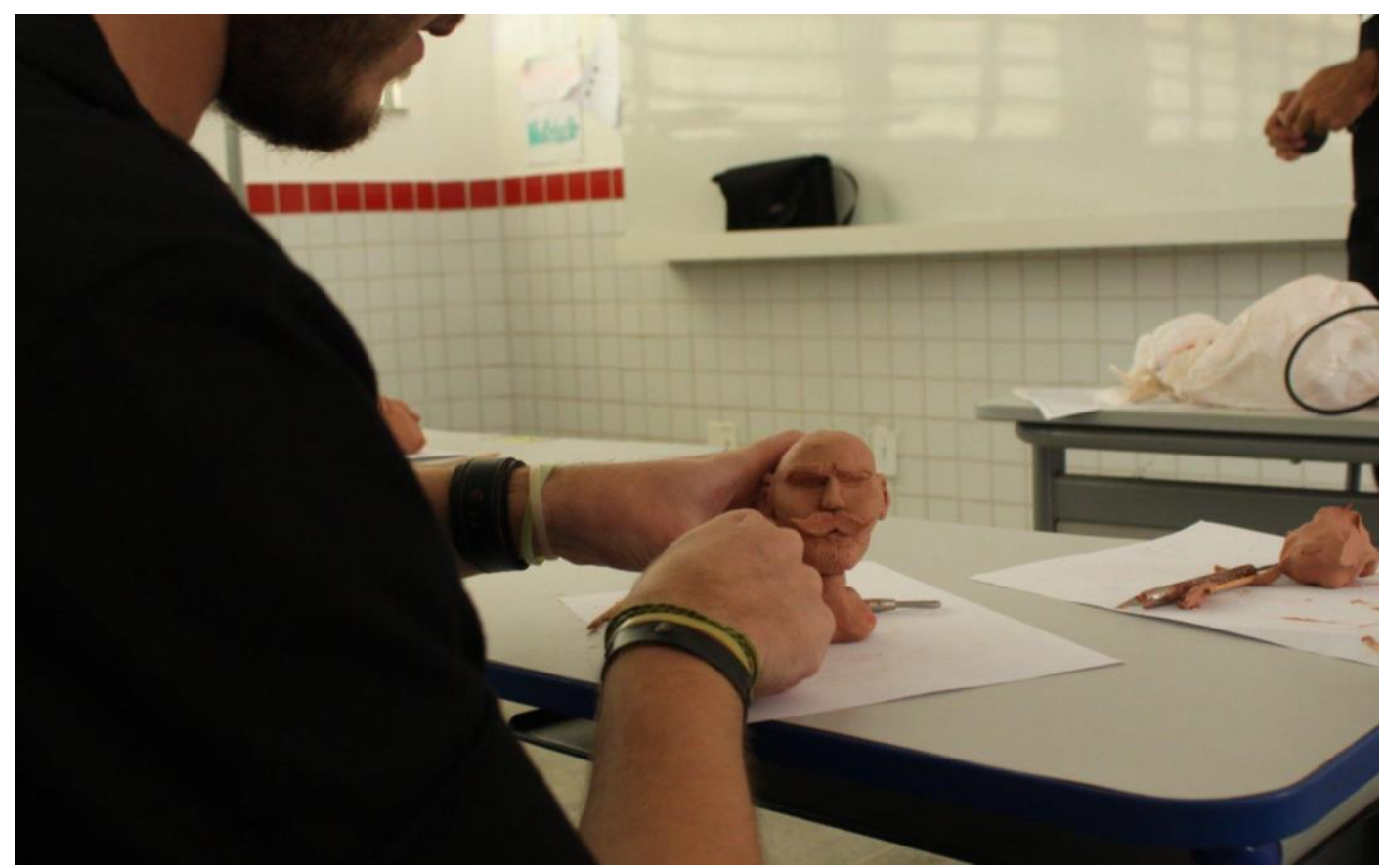

Figura 4 - Explorando a criatividade

Existindo a possibilidade da replicação dos modelos no meio digital, novas abordagens são necessárias. Recomenda-se o uso dos softwares Blender ou Zbrush. Primeiramente é necessário familiarizar os alunos com os princípios básicos da ferramenta. Existem modelos anatômicos tridimensionais prontos e disponibilizados de forma gratuita na internet em sites como Turbosquid2. Utilizar esses modelos representa uma vantagem por dois motivos. Primeiramente, por permitir que, caso o tutor presente não tenha familiaridade com esses softwares de modelagem 3D, possa usar os próprios modelos para estimular a interação e exploração dos alunos, visto que é possível encontrar opções com diferentes níveis de complexidade e fidelidade com a figura humana. Outra vantagem é que, sendo o tutor capaz de orientar os alunos a trabalhar tais softwares de maneira satisfatória, ter um modelo como base acelera significativamente o processo de construção dos personagens no meio digital, sendo necessárias apenas adaptações na forma do modelo.

\section{Resultados e Discussão}

A oficina já foi realizada em 4 EEEPs localizadas em cidades do Ceará nos anos de 2018 e 2019, sendo elas Beberibe, Sobral, Trairi e Nova Russas. Tendo como público alvo alunos do ensino médio. A quantidade de participantes variou entre 15 e 33 pessoas.

Como principal resultado temos a aquisição do conhecimento da nomenclatura científica, da representação bidimensional e tridimensional de cada parte da estrutura do corpo humano proposta na oficina. Esse fato é observado ainda durante o processo, quando havendo dúvidas sobre alguma etapa em específico, alunos citam tais nomenclaturas para se referir à parte em questão.

A ludicidade contribui diretamente para esse resultado visto que a maioria dos alunos demonstram empolgação ao longo do processo, considerando a atividade divertida 
e diferente da forma a qual estão habituados. Outro fato que reforça esse cenário é o apego dos alunos à suas obras, evidenciado com postagens do processo e resultado em redes sociais como Instagram, questionamentos sobre a possibilidade de levarem os modelos para casa e pedidos de fotos ao final da oficina.

A experiência é um aprendizado de mão dupla, pois cada oficina em cada cidade proporciona contato com novos alunos e não raramente agrega novos conhecimentos, possibilidades e até mesmo técnicas. A valorização do conhecimento prévio do aluno é propiciada pois a modelagem manual é um processo que não possui apenas um modo específico de se chegar a determinada representação.

É importante ressaltar o descobrimento pessoal de muitos alunos que não sabiam possuir habilidades manuais até ter o primeiro contato com a modelagem. E para aqueles que já demonstravam interesse pela parte artística do processo, acabam reconhecendo a aquisição do conhecimento teórico sobre anatomia como um bônus. Também vale citar a socialização entre os indivíduos ao longo do processo através da comparação entre as modelos que estão sendo confeccionados bem como ao final com a exposição das obras e troca de opiniões a respeito dos resultados de cada aluno.

É necessário pontuar as dificuldades em realizar a parte digital do processo. A estrutura das escolas nem sempre conta com laboratórios suficientes e disponíveis para comportar diferentes oficinas simultaneamente, visto que ao longo da realização do Projeto Media Day, prioriza-se a disponibilização dos laboratórios existentes para o ensino de conteúdos da área de sistemas, como programação. Porém, existem edições do projeto onde é ofertada a oficina de Modelagem 3D, apresentando-se como possível aliada para o seguimento desta etapa do processo.

Apesar de a oficina ter sido realizada com apenas um público alvo, sua efetividade não se restringe a somente uma faixa etária. Estão documentados resultados positivos na aplicação de métodos semelhantes com alunos universitários do curso de medicina da Universidade do Extremo Sul Catarinense - UNESC no estudo da Anatomia da Embriologia Humana (SILVEIRA e IDERIHA, 2013), além de alunos do ensino fundamental na Unidade Escolar Alexandre Costa, localizada na cidade de Caxias-MA (CARVALHO, 2016). Isso demonstra que uma prática comumente associada ao público infantil e ao ato de brincar pode ser resgatada e ressignificada como ferramenta no processo de aprendizagem.

\section{Conclusão}

A oficina 'Modelagem Manual de Personagens' se apresenta como forma de aprendizado alternativa para conteúdos complexos como Anatomia. Mas é possível expandir sua aplicação para outros campos da ciência que tenham níveis parecidos de complexidade, grande quantidade de conceitos e de difícil visualização, tais como Estrutura e Divisão Celular, Camadas Atmosféricas entre outros.

Além disso, apresenta-se como ferramenta viável para aplicação nas escolas devido ao seu baixo custo e metodologia simples, fatores que costumam ser as principais barreiras na adoção de novos métodos de ensino por parte das escolas. Pode ser oferecida como método complementar às aulas teóricas. Dependendo do nível de complexidade, recomenda-se a capacitação dos professores para que estejam aptos a representar a figura 
anatômica de forma fiel nas primeiras etapas da oficina, visto que seu modelo poderá ser utilizado como referência pelos alunos, além da necessidade de recomendar pequenos ajustes necessários para o refinamento dos modelos produzidos. Não havendo a disponibilidade para a capacitação do professor, a oficina 'Modelagem Manual de Personagens' pode ser aplicada nas escolas interessadas.

Outra vantagem está na possibilidade de replicar os procedimentos do manual para o digital não apenas para visualizar a forma da estrutura em si, mas também seu funcionamento. Através da animação dos modelos tridimensionais incluímos a possibilidade de representar processos morfológicos relacionados às estruturas construídas como por exemplo o batimento de um coração ou o movimento das articulações entre os ossos.

\section{Referências}

AUSUBEL, DAVID P. (1963). A psicologia da aprendizagem verbal significativa. Oxford, Inglaterra: Grune \& Stratton.

AUSUBEL, David P. Educational Pshychology: a cognitive view. 2ed. New York: Holt Rinehart And Winston Inc, 1978. 662 p.

BRAATHEN, Per Christian. Aprendizagem mecânica e aprendizagem significativa no processo de ensino-aprendizagem de Química. Revista Eixo, Brasília, v. 1, n. 1, p.6369, jun. 2012.

CARVALHO, Franciellem de Sousa et al. Utilização de Modelos Didáticos Tridimensionais no Processo de Ensino-aprendizagem de Alunos Surdos e Ouvintes na Disciplina de Ciências. In: Congresso Nacional de Educação, 3., 2016, Natal. Anais... . Natal: Conedu, 2016. p. 1 - 5.

DALGARNO, B. (2002). The Potential of 3D virtual learning environments: A constructivist analysis. Australasian Journal of Educational Technology, 5(2), 1-19

FROSCH, R. Utilização de fabricação digital com modelos 3D para apoio pedagógico à deficientes visuais.. In: ANPED REGIONAL SUDESTE, 2018, CAMPINAS. 13a REUNIÃO CIENTÍFICA DA ANPED. Rio de Janeiro: Anped, 2018. v. I.

LUCKESI, Cipriano Carlos. Educação, Ludicidade e Prevenção das Neuroses Futuras: uma proposta pedagógica a partir da Biossíntese. 2016. Disponível em: $<$ http://luckesi002.blogspot.com/2016/12/educacao-ludicidade-e-prevencaodas_58.html>.Acesso em: 2 dez. 2016.

MCINERNEY, Dennis \& MCINERNEY, V (1994) Educational Psychology Constructing Learning Sydney: Prentice Hall.

MICHAELIS. Dicionário Brasileiro da Língua Portuguesa. 2015. Disponível em: $<$ https://michaelis.uol.com.br/moderno-portugues/busca/portuguesbrasileiro/tecnologia/>. Acesso em: 29 jun. 2019.

MOORE, P. (1995). Learning and teaching in virtual worlds: Implications of virtual reality for education. Australian Journal of Educational Technology, 11(2).

SALBEGOI, Cléton et al. Percepções Acadêmicas sobre o Ensino e a Aprendizagem em Anatomia Humana. Revista Brasileira de EducaÇÃo MÉdica, Brasília, v. 39, n. 1, p.23-31, jan. 2015.

SANT'ANNA, Alexandre; NASCIMENTO, Paulo Roberto do. A história do lúdico na 
educação. Revemat, Florianópolis, v. 06, n. 2, p.19-36, 2011.

SILVEIRA, Sheila Recepute; IDERIHA, Nilce Marzola. Uso de modelo tridimensional de argila no ensino-aprendizagem de Embriologia Humana. In: SEMINÁRIO UNESC DE HUMANIDADES MÉDICAS, 1., 2013, Colatina. Proceedings... . Colatina: Mirabilia Medicinæ, 2013. p. 47 - 55.

SLAVIN, Robert E (1994) Educational Psychology Theory and Practice Allyn and Bacon, Boston. 